\title{
Entwicklung eines Screening-Verfahrens zur Diagnostik von Persönlichkeitsstörungen: Das Persönlichkeitsstörungs-Screening - Kurzform (PSS-K)
}

\author{
Henning Schöttke ${ }^{a} \quad$ Julia Lange ${ }^{a} \quad$ Mario Imholz $^{b} \quad$ Karl Heinz Wiedl ${ }^{a}$ \\ ${ }^{a}$ Poliklinische Psychotherapieambulanz, Institut für Psychologie, Klinische Psychologie und Psychotherapie, Universität Osnabrück,
}

${ }^{\mathrm{b}}$ Ameos Klinikum Osnabrück, Deutschland

\section{Schlüsselwörter \\ Persönlichkeitsstörungen · Diagnostik · Screening · SKID-II}

\section{Zusammenfassung}

Hintergrund: Die Diagnostik von Persönlichkeitsstörungen sollte fester Bestandteil klinischer Eingangsuntersuchungen sein. In der vorliegenden Arbeit wird mit dem Persönlichkeitsstörungs-Screening - Kurzform (PSS-K) ein Screening-Fragebogen vorgestellt, der im sogenannten 2-stufigen Diagnostikprozess im ersten Schritt zur "Schnellerkennung" einer Persönlichkeitsstörung eingesetzt werden kann. Patienten und Methode: Zur Validierung wurden eine nichtklinische Kontrollstichprobe aus der Normalbevölkerung ( $n=392$ ), Insassen einer Justizvollzugsanstalt ( $n=48$ ) und 3 klinische Stichproben rekrutiert: Patienten mit Substanzmissbrauch ( $n=90)$, ambulante Psychotherapiepatienten ( $n=43$ ) und stationäre Psychiatriepatienten ( $n=52$ ). Das SKID-II (Structured Clinical Interview for DSM-IV Personality Disorders) diente bei der Validierung als Goldstandard. Ergebnisse: Das PSS-K erwies sich als reliabel und konstruktvalide. Die Probanden aus der Normalbevölkerung unterscheiden sich von den Testpersonen der anderen Stichproben. Die konvergente Validität der PSS-K-Items und der PSS-KSumme mit den Fragebogen-Scores des SKID-II ist gegeben. Laut Receiver Operating Characteristic (ROC)-Analyse wird bei einem Cut-off-Wert von $>4$ in 93\% der Fälle das Vorliegen mindestens einer Persönlichkeitsstörung richtig erkannt. Schlussfolgerung: Die Reliabilität und Validität des PSS-K konnte mit ersten Ergebnissen belegt werden.

\section{Keywords}

Personality disorders · Assessment - Screening . SCID-II

\section{Summary}

Development of a Screening Measure for the Assessment of Personality Disorders: The Personality Disorder Screening - Short Version (PSS-K)

Background: The assessment of personality disorders should be routine in psychotherapeutic treatment settings. This report describes a new screening measure which could be used as a screening tool in the first step of a 2-stage diagnostic process for personality disorders: the Personality Disorder Screening - Short Version (PSS-K). Patients and Method: To validate the instrument, a non-clinical sample $(n=392)$, prisoners $(n=48)$ and 3 clinical samples, i.e., patients with substance abuse ( $n=90)$, psychotherapeutic outpatients ( $=43$ ) and psychiatric inpatients $(n=52$ ), were recruited. The Structured Clinical Interview for DSM-IV Personality Disorders (SCID-II) was used as the gold standard. Results: The PSS-K appears to be a suitable screening instrument for personality disorders. The non-clinical sample differed significantly from the other subsamples. The items of the PSS-K and the PSS-K score show good convergent validity with the SCID-II questionnaire scores. Using receiver operating characteristic (ROC) analysis, a cut-off value of $>4$ on the screening measure correctly identified the presence of at least one personality disorder in $93 \%$ of cases. Conclusion: Considering the first results, the PSS-K seems to be a reliable and valid screening tool.

\section{KARGER \\ Fax +497614520714 \\ Information@Karger.de}

www.karger.com (c) 2011 S. Karger GmbH, Freiburg

$1016-6262 / 11 / 0213-0154 \$ 38.00 / 0$

Accessible online at:

www.karger.com/ver
Prof. Dr. Henning Schöttke

Universität Osnabrück, Institut für Psychologie

Klinische Psychologie und Psychotherapie, Poliklinische Psychotherapieambulanz

Knollstr. 15, 49069 Osnabrück, Deutschland

Tel. +49 541 9694-757, Fax -028

henning.schoettke@uos.de 


\section{Einleitung}

Die Prävalenz für das Vorliegen einer Persönlichkeitsstörung (PS) liegt in der Allgemeinbevölkerung zwischen 7 und $15 \%$ [Johnson et al., 2008]. In klinischen Behandlungseinrichtungen wird diese Diagnose in 45-66\% der Fälle vergeben [Thuo et al., 2008]. Dementsprechend häufig (13-81\%) ist auch die Komorbidität von PS mit symptomatischen Störungen des Diagnostic and Statistic Manual of Mental Disorders (DSM; sogenannte Achse-I-Störungen) zu beobachten [Dolan-Sewell et al., 2001]. Das Vorliegen einer PS kann sich jedoch ungünstig auf den Behandlungsverlauf der Achse-I-Störung auswirken [Kasen et al., 2007; NewtonHowes et al., 2006; zur Ausnahme siehe Fricke et al., 2003]. Folglich sollte eine reliable und valide Diagnostik von PS fester Bestandteil klinisch-psychometrischer Eingangsdiagnostik sein. Auch wenn die sich anschließende Behandlung nur die symptomatische Störung zum Gegenstand hat, können sich PS-spezifische Interaktionsmerkmale des Patienten hinderlich auf die Durchführung einer symptomorientierten Therapie auswirken. Fragebögen zur Diagnostik von PS sind z.B. das Millon Clinical Multiaxial Inventory (MCMI) [Millon et al., 1997] oder der Personality Disorder Questionnaire-4 (PDQ-4) [Hyler, 1994]. Zudem gibt es Interviewverfahren zur Diagnostik von PS, z.B. das International Personality Disorder Examination (IPDE) [Loranger, 1999], das Structured Clinical Interview for DSM-IV Personality Disorders (SCID-II) [First et al., 1995] oder das Structured Interview for DSM Personality-IV (SIDP-IV) [Pfohl et al., 1995]. Im deutschsprachigen Raum wird auch die Internationale Diagnosen Checkliste für Persönlichkeitsstörungen (IDCL-P) [Bronisch et al., 1995] genutzt.

Die genannten Interviews decken zumindest die Kriterien der im DSM (Versionen III und IV) aufgeführten PS ab und ermöglichen aufgrund ihrer hohen Strukturiertheit eine differenzierte Analyse dieser Störungen. Im deutschsprachigen Raum stellt das SKID-II den aktuellen Goldstandard für die Diagnostik einer PS dar. Die genannten Instrumente sind allerdings sehr umfangreich, zeitlich aufwändig oder verlangen in ihrer Anwendung gut geschultes Personal, wodurch deren Nutzung im Diagnostikprozess sehr zeit- und auch kostenintensiv ist.

Eine Behebung dieses Mankos klinisch-psychologischer Standarddiagnostik könnte nach unserer Auffassung dadurch erreicht werden, dass die Diagnostik von PS adaptiv und gegebenenfalls 2-stufig erfolgt [Mann et al., 1999]: Im ersten Schritt wird ein kurzes valides Screening-Verfahren genutzt, das anzeigt, ob genügend Evidenz für das Vorliegen einer PS vorhanden ist. Dies ist vergleichbar mit der Forderung des DSM-IV oder ICD-10 (International Classification of Diseases), wonach vor einer Spezifizierung der Diagnose erst die allgemeinen Kriterien für das Vorliegen einer PS erfüllt sein müssen [z.B. Fiedler, 2001]. Erst dann würde gegebenenfalls in einem zweiten Schritt mithilfe eines strukturierten Inter- viewverfahrens und/oder weiterer Fragebogenverfahren die Verdachtsdiagnose verifiziert und gegebenenfalls spezifiziert werden. Dieses effektive Vorgehen könnte die klinische Standarddiagnostik optimieren.

\section{Screening-Verfahren für Persönlichkeitsstörungen}

Erste Versuche zur Schnellerkennung einer PS im Diagnostikprozess gingen dahin, den Gesamtwert eines Fragebogens (z.B. PDQ) oder des Screening-Fragebogens, der dem SKIDII-Interview vorgeschaltet ist, zu nutzen [Langbehn et al., 1999]. Die Sensitivität dieser als Screening genutzten Instrumente liegt zwischen 0,35 und 1,00; die Spezifität beträgt 0,43-0,94 [Langbehn et al., 1999]. Die so genutzten Fragebögen sind jedoch sehr lang und somit zeitintensiv in der Anwendung, womit sie für den Einsatz als kurzes ScreeningVerfahren im ersten Schritt des 2-stufigen Diagnostikprozesses ungeeignet sind.

Daraufhin wurden kurze, bislang nur auf Englisch erhältliche und überprüfte Interviews entwickelt, indem man spezifische Items aus den oben genannten Interviewverfahren $\mathrm{zu}$ einem kurzen Screening zusammenfasste [Morse und Pilkonis, 2007]. $\mathrm{Zu}$ diesen Kurzinterviews gehören das Iowa Personality Disorder Screen (IPDS) [Langbehn et al., 1999], das Rapid Personality Assessment Schedule (PAS-R) [Van Horn et al., 2000], die Standardized Assessment of Personality Abbreviated Scale (SAPAS) [Moran et al., 2003] und ein Interview, das auf der Grundlage von 15 DSM-Kriterien entwickelt wurde [Nurnberg et al., 2000]. Die Sensitivität dieser Screening-Interviews liegt zwischen 0,64 und 0,94, die Spezifität zwischen 0,79 und 0,85 . Des Weiteren gibt es einen Kurzfragebogen des Inventory of Interpersonal Problems (IIP-PD), bestehend aus 28 Items [Pilkonis et al., 1996], der als Screening für PS genutzt wird (Sensitivität 0,71-0,91, Spezifität 0,40-0,67). Die Nachteile dieser Verfahren sind darin zu sehen, dass entweder keine überprüften deutschsprachigen Versionen zur Verfügung stehen, die Kurzversionen immer noch zu lang sind oder die psychometrischen Kennwerte der Verfahren eine zu große Zahl von Fehlklassifikationen bedingen.

In dieser Studie wird ein neuer Screening-Fragebogen vorgestellt, die Kurzform des Persönlichkeitsstörungs-Screenings (PSS-K) (Abb. 1). Ziel dieser Neuentwicklung ist es, ein kurzes, deutschsprachiges, ökonomisch anwendbares und sensitives Screening-Instrument zur Verfügung zu stellen. Anhand des PSS-K-Gesamtwertes soll im ersten Schritt eines optimierten Diagnostikprozesses, analog zur Überprüfung der allgemeinen Kriterien für das Vorliegen einer PS im DSM-IV und ICD-10, beurteilt werden, ob in einem zweiten Schritt eine nachfolgende differenzierte Diagnostik indiziert ist.

Im Folgenden wird die Testentwicklung des PSS-K beschrieben und die Konstruktvalidierung am SKID-II dargestellt. Zudem wird ein Cut-off-Wert ermittelt, der Personen mit einer PS, diagnostiziert nach dem SKID-II, optimal von Gesunden trennt. 


\section{Der Fragebogen zum Persönlichkeitsstörungs-Screening - Kurzform (PSS-K)}

Bitte lesen Sie jede Feststellung durch. Kreuzen Sie bitte das Antwortkästchen der Feststellung an, die am besten auf Sie zutrifft.

Manche Feststellung besteht aus zwei Teilen. Wenn Sie nur mit einem Teil einverstanden sind, kreuzen Sie bitte Vielleicht an; wenn Sie mit beiden Teilen einverstanden sind, kreuzen Sie bitte Ja an. Lassen Sie bitte keine Feststellung aus, auch wenn Sie meinen, eine Feststellung träfe auf Sie oder Ihre Lebensumstände nicht zu; antworten Sie bitte so, als würde sie zutreffen.

\begin{tabular}{|c|c|c|c|c|}
\hline Nr. & & $\mathrm{Ja}$ & Vielleicht & Nein \\
\hline 1 & $\begin{array}{l}\text { Ich mache mir sehr viele Sorgen, dass } \\
\text { Menschen, an denen mir etwas liegt, } \\
\text { mich verlassen, obwohl gewöhnlich kein } \\
\text { Grund für diese Angst besteht. }\end{array}$ & $\square$ & $\square$ & $\square$ \\
\hline 2 & $\begin{array}{l}\text { Ich kann ungeduldig sein; im Allgemeinen } \\
\text { will ich das, was ich will, sofort. }\end{array}$ & $\square$ & $\square$ & $\square$ \\
\hline 3 & $\begin{array}{l}\text { Im Allgemeinen habe ich sehr intensive } \\
\text { Beziehungen und gewöhnlich schwanken } \\
\text { meine Gefühle für diesen Menschen von } \\
\text { einem Extrem zum anderen. Manchmal } \\
\text { bete ich ihn fast an und dann wieder kann } \\
\text { ich ihn nicht ausstehen. }\end{array}$ & $\square$ & $\square$ & $\square$ \\
\hline 4 & $\begin{array}{l}\text { Ich habe nicht besonders viel Vertrauen, } \\
\text { obwohl ich es gern hätte. Ich habe einfach } \\
\text { Angst, dass Menschen mich ausnutzen } \\
\text { könnten, wenn ich nicht vorsichtig bin. }\end{array}$ & $\square$ & $\square$ & $\square$ \\
\hline 5 & $\begin{array}{l}\text { Ich neige dazu, ein Einzelgänger zu sein, } \\
\text { und für mich ist das in Ordnung. Es macht } \\
\text { mir irgendwie keinen Spaß, viel mit } \\
\text { anderen Leuten zusammen zu sein, auch } \\
\text { wenn es meine Familie ist. }\end{array}$ & $\square$ & $\square$ & $\square$ \\
\hline 6 & $\begin{array}{l}\text { Ich bin sehr unsicher. Ich habe oft das } \\
\text { Gefühl, dass Menschen mich ansehen } \\
\text { und mich taxieren, nicht immer in } \\
\text { schmeichelhafter Weise. }\end{array}$ & $\square$ & $\square$ & $\square$ \\
\hline 7 & $\begin{array}{l}\text { Ich bleibe lieber bei meiner üblichen } \\
\text { täglichen Routine als mich in unbekannte } \\
\text { Umgebungen und Situationen zu wagen. }\end{array}$ & $\square$ & $\square$ & $\square$ \\
\hline 8 & $\begin{array}{l}\text { Mich fasziniert eine Art Untergrundleben, } \\
\text { in dem man die Regeln brechen kann und } \\
\text { ungestraft davonkommt. }\end{array}$ & $\square$ & $\square$ & $\square$ \\
\hline
\end{tabular}

Abb. 1. Der Fragebogen zum Persönlichkeitsstörungs-Screening - Kurzform (PSS-K). 


\section{Methoden}

Das PSS-K basiert auf einem Fragebogen zur dimensionalen Diagnostik von Persönlichkeitsstilen und -störungen, dem Persönlichkeitsselbstportrait (PSP), entwickelt von Oldham und Morris [1990, 2008]. Das Persönlichkeitsstörungs-Screening (PSS) wurde aus der von Oldham und Morris [1992] berichteten deutschen Übersetzung des PSP an der Poliklinischen Psychotherapieambulanz der Universität Osnabrück entwickelt. Angaben zu Gütekriterien der deutschen und der englischen Version des PSP liegen bislang nicht vor.

Für die Entwicklung des PSS wurden nur 10 der ursprünglich 13 Skalen des PSP verwendet. Berücksichtigt wurden die korrespondierenden Skalen der 10 Persönlichkeitsstörungen des DSM-IV. Aus den jeweiligen Items dieser 10 Skalen wurden stellvertretend für jede PS 10 Items ausgewählt, welche die höchste Trennschärfe zu ihrer entsprechenden Subskala aus dem PSP aufwiesen. Der Zielsetzung folgend, ein möglichst kurzes Instrument zu entwickeln, wurde diese erste Screening-Version in einem zweiten Entwicklungsschritt auf eine Kurzform mit 8 Items (PSS-K) reduziert. Ausgewählt wurden die Items mit der höchsten Effektstärke, berechnet nach Hedges und Olkin [1985, zit. nach Rustenbach, 2003], die am besten zwischen dem Vorliegen mindestens einer versus keiner PS, diagnostiziert nach dem SKID-II, differenzierten. Die verbleibenden Items repräsentieren die Skalen dependent (PSS-K1), histrionisch (PSS-K2), borderline (PSS-K3), paranoid (PSS-K4), schizoid (PSS-K5), schizotypisch (PSS-K6), zwanghaft (PSS-K7) und antisozial (PSS-K8). Die Itemwerte der Antwortmöglichkeiten (Nein = 0, Vielleicht $=1, \mathrm{Ja}=2$ ) werden zum PSS-K-Gesamtwert aufsummiert

Die Konstruktvalidierung des PSS-K erfolgte am SKID-II [Fydrich et al., 1997]. Das SKID-II ist ein 2-stufiges Verfahren, bestehend aus einem Fragebogen mit 119 Items und einem Interview. Jedes mit Ja beantwortete Item wird im anschließenden Interview nachexploriert und dahingehend bewertet ob die Kriterien für das Vorliegen einer PS erfüllt sind. In dieser Studie wurde zudem der dimensionale Fragebogen-Score (FB-Score) des SKID-II zur Validierung des PSS-K genutzt. Die Reliabilität und Validität des SKID-II werden als gut beschrieben; für eine ausführliche Darstellung sei auf Smith et al. [2003] verwiesen.

\section{Stichproben}

In die vorliegende Studie gehen verschiedene Stichproben ein, die in unterschiedlichen Studienprojekten gewonnen wurden [Schöttke, 2006]. Das Versuchsprotokoll wurde vom Ethikbeauftragten des Instituts genehmigt. Die Probanden der Untersuchung wurden über die Studie aufgeklärt und nahmen freiwillig teil. Es wurden insgesamt 625 Personen untersucht. Neben Probanden aus der Normalbevölkerung wurden klinische Stichproben und eine weitere rekrutiert, weil entweder hohe Prävalenzen von PS zu vermuten waren oder die Diagnose einer PS aus therapeutischen oder gutachterlichen Gründen von Interesse sein könnte. Die nichtklinischen Stichproben setzen sich aus einer unausgelesenen Stichprobe der Normalbevölkerung $(\mathrm{n}=392)$ sowie einer Stichprobe Strafgefangener aus einer Justizvollzugsanstalt (JVA; $n=48$ ) [zur Bedeutung von PS bei Strafgefangenen siehe Frädrich und Pfäfflin, 2000] zusammen. Bei der Auswahl der Stichprobe aus der Normalbevölkerung wurden nur Probanden berücksichtigt, die seit einem Jahr keine psychotherapeutische oder psychopharmakologische Behandlung in Anspruch genommen hatten. Die Strafgefangenen der Stichprobe waren im Durchschnitt $\mathrm{zu}$ einer Haftstrafe von 3,9 Jahren (Standardabweichung $(\mathrm{SD})=2,46$ Jahre) verurteilt. Zu den häufigsten Straftaten gehörten Körperverletzung oder Totschlag $(\mathrm{n}=12)$, Einbruch, Diebstahl oder Raub $(\mathrm{n}=9)$ und sexuelle Delikte $(\mathrm{n}=8)$. Zu den klinischen Stichproben gehörten Patienten mit Störungen des Substanzgebrauchs (vornehmlich illegale Drogen und Alkohol), die sich zwecks einer Entgiftungsbehandlung in stationärer Behandlung befanden $(\mathrm{n}=90)$ [zur Bedeutung von PS für Drogenkonsumenten siehe Hesse et al., 2008], Patienten der Poliklinischen Psychotherapieambulanz der Universität Osnabrück $(n=43)$ und Patienten aus

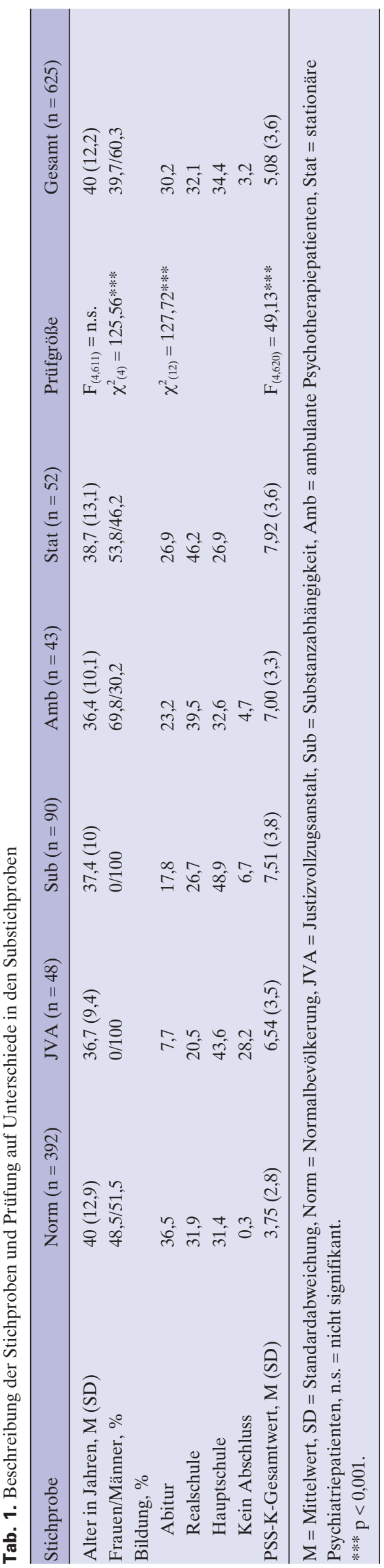


Tab. 2. Itemkennwerte in den verschiedenen Substichproben und in der Gesamtstichprobe

\begin{tabular}{|c|c|c|c|c|c|c|c|}
\hline \multicolumn{2}{|c|}{ Item (PSS-K) } & \multicolumn{6}{|l|}{ Stichprobe } \\
\hline & & $\operatorname{Norm}(n=392)$ & JVA $(n=48)$ & $\operatorname{Sub}(n=90)$ & $\operatorname{Amb}(n=43)$ & Stat $(\mathrm{n}=52)$ & Gesamt $(n=625)$ \\
\hline \multirow[t]{3}{*}{1} & $\mathrm{M}(\mathrm{SD})$ & $0,4(0,7)$ & $1,0(1,0)$ & $1,2(0,9)$ & $1,3(0,8)$ & $1,2(0,9)$ & $0,7(0,9)$ \\
\hline & $\mathrm{Pi}$ & 21 & 48 & 58 & 63 & 61 & 35 \\
\hline & $\mathrm{r}_{\mathrm{it}}$ & 0,4 & 0,3 & 0,5 & 0,4 & 0,3 & 0,5 \\
\hline \multirow[t]{2}{*}{2} & $\mathrm{M}(\mathrm{SD})$ & $1,0(0,9)$ & $1,2(0,9)$ & $1,3(0,9)$ & $1,2(0,9)$ & $1,3(0,8)$ & $1,1(0,9)$ \\
\hline & $\mathrm{r}_{\mathrm{it}}$ & 0,1 & 0,2 & 0,4 & 0,4 & 0,4 & 0,3 \\
\hline \multirow[t]{3}{*}{3} & $\mathrm{M}(\mathrm{SD})$ & $0,4(0,7)$ & $0,5(0,8)$ & $0,9(0,9)$ & $0,7(0,9)$ & $0,9(0,8)$ & $0,5(0,8)$ \\
\hline & $\mathrm{Pi}$ & 20 & 24 & 47 & 34 & 42 & 27 \\
\hline & $\mathrm{r}_{\mathrm{it}}$ & 0,4 & 0,4 & 0,4 & 0,2 & 0,3 & 0,4 \\
\hline \multirow[t]{2}{*}{4} & $\mathrm{M}(\mathrm{SD})$ & $0,4(0,7)$ & $1,5(0,7)$ & $1,0(0,9)$ & $1,1(0,9)$ & $1,1(0,8)$ & $0,7(0,8)$ \\
\hline & $\mathrm{Pi}$ & 20 & 76 & 56 & 57 & 53 & 36 \\
\hline \multirow[t]{3}{*}{5} & $\mathrm{M}(\mathrm{SD})$ & $0,3(0,6)$ & $0,6(0,8)$ & $0,7(0,8)$ & $0,4(0,6)$ & $0,8(0,9)$ & $0,4(0,7)$ \\
\hline & $\mathrm{Pi}$ & 15 & 30 & 36 & 22 & 38 & 22 \\
\hline & $\mathrm{r}_{\mathrm{it}}$ & 0,3 & 0,2 & 0,4 & 0,2 & 0,3 & 0,4 \\
\hline \multirow[t]{3}{*}{6} & $\mathrm{M}(\mathrm{SD})$ & $0,3(0,6)$ & $0,7(0,9)$ & $0,7(0,8)$ & $0,9(0,9)$ & $1,2(0,9)$ & $0,5(0,8)$ \\
\hline & $\mathrm{Pi}$ & 15 & 36 & 34 & 47 & 61 & 25 \\
\hline & $\mathrm{r}_{\mathrm{it}}$ & 0,4 & 0,4 & 0,5 & 0,3 & 0,7 & 0,5 \\
\hline \multirow[t]{3}{*}{7} & $\mathrm{M}(\mathrm{SD})$ & $0,7(0,8)$ & $0,5(0,8)$ & $0,6(0,8)$ & $1,0(0,8)$ & $1,1(0,9)$ & $0,7(0,8)$ \\
\hline & $\mathrm{Pi}$ & 34 & 26 & 32 & 50 & 56 & 36 \\
\hline & $\mathrm{r}_{\mathrm{it}}$ & 0,3 & 0,4 & 0,3 & 0,3 & 0,3 & 0,3 \\
\hline \multirow[t]{3}{*}{8} & $\mathrm{M}(\mathrm{SD})$ & $0,3(0,6)$ & $0,5(0,8)$ & $0,9(0,8)$ & $0,3(0,6)$ & $0,4(0,8)$ & $0,4(0,7)$ \\
\hline & $\mathrm{Pi}$ & 15 & 26 & 46 & 16 & 21 & 21 \\
\hline & $\mathrm{r}_{\mathrm{it}}$ & 0,1 & 0,4 & 0,1 & 0,1 & 0,1 & 0,2 \\
\hline
\end{tabular}

Norm = Normalbevölkerung, JVA = Justizvollzugsanstalt, Sub = Substanzabhängigkeit, Amb = ambulante Psychotherapiepatienten

Stat = stationäre Psychiatriepatienten, $M=$ Mittelwert, $\mathrm{SD}=$ Standardabweichung, $\mathrm{Pi}=\mathrm{Schwierigkeit}, \mathrm{r}_{\mathrm{it}}=$ Trennschärfe .

einer Fachklinik für Psychiatrie $(\mathrm{n}=52)$ [zur Bedeutsamkeit von PS im psychiatrischen und psychotherapeutischen Bereich siehe Lamont und Brunero, 2009; Samuels et al., 2002]. Eine Kopie der von den Patienten bzw. Gefängnisinsassen unterschriebenen Erklärung zur Aufklärung wurde in den Patienten- oder JVA-Akten hinterlegt. Tabelle 1 gibt einen Überblick über Alter, Geschlechterverteilung und das Bildungsniveau in der Gesamtstichprobe und den Substichproben.

Die Substichproben unterscheiden sich hinsichtlich der Geschlechterverteilung und des Bildungsstandes. Zudem zeigen sich signifikante Unterschiede für den PSS-K-Gesamtwert in den Substichproben. Für die Stichprobe aus der Normalbevölkerung ergibt sich der geringste Wert und für die Stichprobe aus der Psychiatrie der höchste. Die Stichprobe aus der Normalbevölkerung weist deskriptiv den höchsten Bildungsstand auf, die JVA-Stichprobe den niedrigsten.

Die Hauptdiagnosen in der Stichprobe der ambulanten Psychotherapiepatienten bestehen nach dem ICD-10 aus affektiven Störungen (F3X; $55,8 \%$ ), neurotischen, Belastungs- und somatoformen Störungen (F4X; $55,8 \%$ ) und Essstörungen (F50.X; 16,3\%). Die Patienten weisen im Durchschnitt 1,63 Störungsdiagnosen auf. In der Stichprobe der stationären Psychiatriepatienten zeigen sich folgende Hauptdiagnosen nach dem ICD-10: affektive Störungen (F3X; 71,2\%), neurotische, Belastungs- und somatoforme Störungen (F4X; 19,2\%) und Essstörungen (F50.X; 7,7\%). Diese Patienten weisen im Durchschnitt 1,73 Störungsdiagnosen auf. Die Gruppe der Suchtpatienten bestand aus $n=40$ Patienten mit Polytoxikomanie (ICD-10: F19,2), aus n = 40 Patienten mit Alkoholabhängigkeit (ICD-10: F10,2) und n = 10 Patienten, die neben ihrer Alkoholabhängigkeit von mindestens einer illegalen Droge abhängig waren (ICD-10: F10,2 und F1X.2).
Alle 625 Personen, deren Daten in diese Studie eingehen, bearbeiteten das PSS-K. Die Daten der Stichproben aus der Normalbevölkerung, der JVA und der Suchtpatienten dienten dabei der Testentwicklung und Normierung, die zeitlich spätere Validierung am SKID-II erfolgte mit den ambulanten Psychotherapie- und Psychiatriepatienten.

\section{Ergebnisse}

\section{Verteilungskennwerte und Reliabilität}

In Tabelle 2 sind die Mittelwerte, Standardabweichungen, Schwierigkeiten und Trennschärfen der PSS-K-Items für die Substichproben und die Gesamtstichprobe dargestellt.

Betrachtet man zunächst die Itemschwierigkeiten, sind die PSS-K-Items 5 und 8 am schwierigsten, das PSS-K-Item 2 am leichtesten. Die Trennschärfen sind zum größten Teil zufriedenstellend, mit Ausnahme des PSS-K-Items 8, bei dem die Trennschärfe nahezu stichprobenübergreifend gering ist. Die interne Konsistenz des PSS-K beträgt $\alpha=0,69$ für die Gesamtstichprobe. Die Reliabilitäten der Substichproben variieren entsprechend der unterschiedlichen Anzahl der untersuchten Probanden (Normalbevölkerung $\alpha=0,58$; Gefängnisinsassen $\alpha=0,63$; Suchtpatienten $\alpha=0,68$; ambulante Psychotherapiepatienten $\alpha=0,60$; stationäre Psychiatriepatienten $\alpha=0,64$ ). 
Tab. 3. Interkorrelation der Items des PSS-K $(n=625)$ und Korrelation mit der Anzahl der Persönlichkeitsstörungen nach SKID-II $(n=95$, Stichproben Amb, Stat)

\begin{tabular}{|c|c|c|c|c|c|c|c|c|c|}
\hline & PSS-K1 & PSS-K2 & PSS-K3 & PSS-K4 & PSS-K5 & PSS-K6 & PSS-K7 & PSS-K8 & Anzahl SKID-II \\
\hline PSS-K1 & 1,00 & $0,21 * *$ & $0,35 * *$ & $0,46 * *$ & $0,17 * *$ & $0,40 * *$ & $0,21 * *$ & $0,11 * *$ & $0,23^{*}$ \\
\hline PSS-K2 & & 1,00 & $0,25 * *$ & $0,15 * *$ & $0,13 * *$ & $0,21 * *$ & 0,05 & $0,15^{* *}$ & $0,32 * *$ \\
\hline PSS-K3 & & & 1,00 & $0,28 * *$ & $0,11 * *$ & $0,25 * *$ & $0,11 * *$ & $0,24 * *$ & $0,27 * *$ \\
\hline PSS-K4 & & & & 1,00 & $0,31 * *$ & $0,43 * *$ & $0,25 * *$ & $0,10^{*}$ & $0,24^{*}$ \\
\hline PSS-K5 & & & & & 1,00 & $0,34 * *$ & $0,25^{* *}$ & $0,18 * *$ & $0,25^{*}$ \\
\hline PSS-K6 & & & & & & 1,00 & $0,29 * *$ & $0,11 * *$ & $0,40 * *$ \\
\hline PSS-K7 & & & & & & & 1,00 & $-0,07$ & $0,28 * *$ \\
\hline PSS-K8 & & & & & & & & 1,00 & $0,22 *$ \\
\hline
\end{tabular}

Tab. 4. Gütekriterien für mögliche Cut-offWerte ( $n=95$, Stichproben Amb, Stat)

\begin{tabular}{llllll}
\hline Cut-off-Wert & Sens & Spez & Youden-Index & PPW & NPW \\
\hline$>0$ & 1 & 0,07 & 0,07 & 0,59 & 1 \\
$>1$ & 1 & 0,15 & 0,15 & 0,61 & 1 \\
$>2$ & 1 & 0,19 & 0,19 & 0,62 & 1 \\
$>3$ & 0,98 & 0,27 & 0,25 & 0,64 & 0,92 \\
$>4$ & 0,93 & 0,44 & 0,37 & 0,68 & 0,82 \\
$>5$ & 0,87 & 0,51 & 0,38 & 0,70 & 0,75 \\
$>6$ & 0,80 & 0,63 & 0,43 & 0,74 & 0,70 \\
$>7$ & 0,74 & 0,68 & 0,42 & 0,75 & 0,67 \\
$>8$ & 0,59 & 0,83 & 0,42 & 0,82 & 0,61 \\
$>9$ & 0,48 & 0,88 & 0,36 & 0,84 & 0,56 \\
$>10$ & 0,33 & 0,93 & 0,26 & 0,86 & 0,51 \\
$>11$ & 0,17 & 0,93 & 0,10 & 0,75 & 0,46 \\
$>12$ & 0,07 & 0,98 & 0,05 & 0,80 & 0,44 \\
$>13$ & 0,04 & 1 & 0,04 & 1 & 0,44 \\
$>14$ & 0,02 & 1 & 0,02 & 1 & 0,44 \\
\hline Sens $=$ Sensitivität, Spez $=$ Spezifität, PPW = positiver prädiktiver Wert, & \\
NPW = negativer prädiktiver Wert. & & & \\
\hline
\end{tabular}

\section{Validität}

Die Interkorrelationen der PSS-K-Items sind in Tabelle 3 dargestellt. Die PSS-K-Items 1, 4 und 6 weisen sehr hohe Zusammenhänge untereinander auf. Die Korrelationen der übrigen Items liegen im unteren bis mittleren Bereich. Die durchschnittliche Iteminterkorrelation beträgt $\bar{r}_{\alpha}=0,21$ (nach Fisher z-Transformation).

\section{Konvergente Validität}

Für die Patienten aus der Stichprobe der ambulanten Psychotherapiepatienten und der stationären Psychiatriepatienten ( $\mathrm{n}=95)$ zeigen sich folgende Prävalenzen der einzelnen PS nach dem SKID-II Interview: selbstunsicher (31,6\%), zwanghaft $(14,7 \%)$, borderline $(11,6 \%)$, paranoid $(6,3 \%)$, dependent $(4,2 \%)$, histrionisch $(4,2 \%)$, narzisstisch $(4,2 \%)$, negativistisch $(3,2 \%)$, antisozial $(3,2 \%)$, schizotypisch $(2,1 \%)$ und schizoid $(1,1 \%)$. Insgesamt 56,8\% der Patienten haben mindestens eine komorbide PS. Im Durchschnitt liegen in dieser Patientenstichprobe 1,14 Diagnosen von PS vor.
Jedes Item des PSS-K korreliert zwischen 0,22 und 0,4 (alle p-Werte < 0,05) mit der Anzahl der PS im SKID-II (Tab. 3). Die PSS-K-Summe und die Anzahl der PS korrelieren zu 0,53 $(\mathrm{p}<0,01)$. Weiter finden sich signifikante bivariate Korrelationen (alle p-Werte $<0,01$ ) zwischen der PSS-K-Summe und allen FB-Scores des SKID-II $(0,34<r>0,56)$, mit Ausnahme der Skalen «antisozial» $(\mathrm{r}=0,18 ; \mathrm{p}<0,10)$ und «histrionisch» ( $\mathrm{r}=0,13$, nicht signifikant (n.s.)).

Die klinische Differenzierungsfähigkeit des PSS-K-Gesamtwertes ist gegeben, da sich die Stichprobe aus der Normalbevölkerung von allen anderen Stichproben unterscheidet (Tab. 1; $\left.\mathrm{F}_{(4,620)}=49,13 ; \mathrm{p}<0,001\right)$. Erwartungsgemäß findet sich für die Probanden aus der Normalbevölkerung der geringste PSS-K-Gesamtwert und für die Psychiatriepatienten der höchste.

\section{Bestimmung eines Cut-off-Wertes}

Die Bestimmung eines Cut-off-Wertes erfolgt mit der Receiver Operating Characteristic (ROC)-Analyse [Murphy et al., 
1987]. Die Fläche unter der ROC-Kurve als globales Maß für die Güte des Tests beträgt 0,79 (95\% Konfidenz-Intervall: 0,70-0,88). In Tabelle 4 sind mögliche Cut-off-Werte und diagnostische Kennwerte angegeben.

Zur Darstellung der Gütekriterien und deren Berechnung werden folgende Abkürzungen benutzt: richtig-positiv (RP), falsch-positiv (FP), richtig-negativ (RN) und falsch-negativ (FN). Die Sensitivität ist ein Maß für die Trefferquote, also die Wahrscheinlichkeit, dass eine erkrankte Person durch einen Test erkannt wird $(\mathrm{RP} /(\mathrm{FN}+\mathrm{RP}))$. Die Spezifität bezeichnet die Quote der korrekten Ablehnungen $(\mathrm{RN} /(\mathrm{FP}+\mathrm{RN}))$. Der positive prädiktive Wert (PPW) gibt die Wahrscheinlichkeit an, mit der bei einem positiven Testergebnis auch tatsächlich eine Erkrankung vorliegt ( RP/(RP + FP)). Der negative prädiktive Wert (NPW) bezeichnet die Wahrscheinlichkeit, mit der bei einem negativen Testergebnis keine Erkrankung vorliegt $(\mathrm{RN} /(\mathrm{RN}+\mathrm{FN}))$. Der Youden-Index wird gebildet durch Sensitivität + Spezifität - 1 und gibt an, für welchen Schwellenwert die Summe von Sensitivität und Spezifität am größten ist und somit die Separierung der Gruppen am besten gelingt [Moosbrugger und Kelava, 2007]. Die Festlegung eines geeigneten Trennwertes bedarf der Abwägung verschiedener Kriterien, worauf in der Diskussion eingegangen wird.

\section{Diskussion}

Ziel der Studie war die Entwicklung eines kurzen und validen Screening-Fragebogens, der im 2-stufigen Diagnostik-Prozess das Vorliegen einer PS anzeigen soll.

Zunächst ist zu diskutieren, ob die Höhe der Reliabilität des PSS-K, geschätzt über die interne Konsistenz von Cronbachs $\alpha$ mit 0,69 , angemessen ist. Folgende Gesichtspunkte sollten bedacht werden: Moosbrugger und Kelava [2007] weisen darauf hin, dass die Reliabilität von Screening-Verfahren aufgrund ihrer geringen Itemzahl im Vergleich zu ausführlichen Verfahren meistens geringer ist und die Reliabilität von einzelnen Skalen gängiger Persönlichkeitstests oft nur im Bereich von 0,70 liegt [Moosbrugger und Kelava, 2007]. Eine Testverlängerung des PSS-K von 8 auf 16 Items würde eine Erhöhung der Reliabilität auf $\alpha=0,82$ bewirken [Moosbrugger und Kelava, 2007]. Zudem stellen PS sehr heterogene Konstrukte dar und werden folglich am besten durch Instrumente erfasst, die aus heterogenen Items bestehen. Dies ist im PSS-K durch eine eher geringe Iteminterkorrelation in Höhe von $\bar{r}_{i t}=0,21$ gegeben. Moosbrugger und Kelava [2007] merken hierzu an, dass die Reliabilitätsbestimmung bei geringer Konsistenz unter Umständen stark unterschätzt wird, wobei inhaltlich heterogene Items die konsistenzanalytisch bestimmte Reliabilität zwar mindern, aber trotzdem eine hohe Vorhersagevalidität erzielen können. Des Weiteren zeigen vergleichbar kurze Screening-Instrumente wie die SAPAS eine ähnlich geringe Reliabilität mit Cronbachs $\alpha=0,68$ [Moran et al., 2003]. Vor diesem Hintergrund ist die Reliabi- lität des PSS-K als ausreichend anzusehen. Für die Zukunft sollte in weiteren Studien die Retest-Reliabilität des PSS-K als ergänzendes $\mathrm{Ma}$ bestimmt werden.

Die Validierung am Goldstandard (SKID-II) ist sehr erfolgversprechend. Der Screening-Gesamtwert und die Einzelitems korrelieren bedeutsam mit der Anzahl der PS und überwiegend mit den dimensionalen FB-Scores des SKID-II. Der PSSK-Gesamtwert sagt das Vorliegen mindestens einer PS nach dem SKID-II valide vorher. Die klinische Differenzierungsfähigkeit des PSS-K konnte belegt werden. Die Substichproben unterscheiden sich signifikant im PSS-K-Gesamtwert.

Bei der Festlegung eines geeigneten Cut-off-Wertes sind folgende Gesichtspunkte zu beachten: Soll das Screening in einem ersten Diagnoseschritt zur Schnellerkennung einer bestimmten Erkrankung eingesetzt werden und wird im Falle eines positiven Ergebnisses im zweiten Schritt eine ausführliche Diagnostik zur Spezifizierung der Erkrankung durchgeführt, dann ist das Hauptziel die Erkennung aller Erkrankten. Hierfür wird eine möglichst hohe Sensitivität gefordert, bei gleichzeitig akzeptabler Spezifität. Folgt man diesem Kriterium, würde man sich für den Schwellenwert $>4$ entscheiden (Sensitivität $=0,93$, Spezifität $=0,44)$. Die Wahrscheinlichkeit, dass ein positives Ergebnis des PSS-K bei diesem Cut-offWert richtig ist, beträgt $68 \%$ (PPW); ein negatives ScreeningErgebnis stimmt in $82 \%$ der Fälle (NPW). Orientiert man sich hingegen bei der Wahl eines geeigneten Cut-off-Wertes am Youden-Index, würde man für den Trennwert von $>6$ plädieren. Bei einer Sensitivität von 0,80 werden $80 \%$ der Erkrankten auch wirklich erkannt, in $20 \%$ der Fälle wird jedoch eine PS übersehen. Der PPW beträgt 0,74 und der NPW 0,70.

Für den Einsatz des PSS-K im 2-stufigen Diagnostikprozess steht bei der Wahl eines geeigneten Cut-off-Wertes nicht die Ausbalancierung von Sensitivität und Spezifität (maximaler Youden-Index) im Vordergrund, sondern eine höchstmögliche Sensitivität (hohe Trefferquote) bei hinreichender Spezifität (nicht zu viele falsch-positive Diagnosen). Bei zusätzlicher Abwägung der Konsequenz einer falsch-negativen Diagnose («Verpasser» - Übersehen einer vorhandenen PS) im Vergleich zur Konsequenz einer falsch-positiven Diagnose («falscher Alarm»- Durchführung einer zusätzlichen Diagnostik) sollte der Cut-off-Wert $>4$ gewählt werden.

Betrachtet man vergleichbare Kennwerte des bislang einzigen existierenden englischen Screening-Fragebogens, des IIP-PD (28 Items; Sensitivität $=0,91$, Spezifität $=0,40$ ) [Pilkonis et al., 1996], sind die ermittelten Screening-Kennwerte des PSS-K als sehr positiv zu beurteilen.

In zukünftigen Untersuchungen zur Validierung des PSS-K ist die Eignung dieses ermittelten Trennwertes in unterschiedlichen Stichproben zu überprüfen.

\section{Disclosure Statement}

Die Autoren erklären, dass kein Interessenkonflikt vorliegt. 


\section{Literatur}

Bronisch T, Hiller W, Mombour W, et al.: Internationale Diagnosen Checkliste für Persönlichkeitsstörungen nach dem ICD-10 und DSM-IV. Bern, Huber, 1995.

Dolan-Sewell RT, Krueger RF, Shea MT: Co-occurrence with syndrome disorders; in Livesley $\mathrm{J}$ (ed) Handbook of Personality Disorders: Theory, Research, and Treatment. New York, The Guilford Press, 2001.

Frädrich S, Pfäfflin F: Zur Prävalenz von Persönlichkeitsstörungen bei Strafgefangenen. Recht Psychiatrie 2000;18:95-104.

Fiedler P: Persönlichkeitsstörungen. Göttingen, Beltz, 2001.

First MB, Spitzer RL, Gibbon M, et al.: The Structured Clinical Interview for DSM-III-R Personality Disorders (SCID-II). Washington, American Psychiatric Press, 1995.

Fricke S, Moritz S, Andresen B, et al.: Einfluss von

Persönlichkeitsstörungen auf den Erfolg einer multimodalen Verhaltenstherapie bei Zwangserkrankungen - Teil I: Grundprobleme. Verhaltenstherapie 2003;13:166-171.

Fydrich T, Renneberg B, Schmitz B, et al.: SKID-II Strukturiertes Klinisches Interview für DSM-IV. Achse-II: Persönlichkeitsstörungen. Interviewheft Göttingen, Hogrefe, 1997.

Hesse M, Rasmussen J, Pedersen MK: Standardised

assessment of personality - a study of validity and reliability in substance abusers. BMC Psychiatry 2008;8:7.

Hyler SE: Personality Diagnostic Questionnaire-4 (PDQ-4+). New York, New York State Psychiatric Institute, 1994.

Johnson JG, Cohen P, Kasen S, et al.: Cumulative

prevalence of personality disorders between adolescence and adulthood. Acta Psychiatr Scand 2008; 118:410-413.

Kasen S, Cohen P, Skodol AE, et al.: Comorbid per-

sonality disorder and treatment use in a community sample of youths: a 20-year follow-up. Acta Psychiatr Scand 2007;115:56-65.
Lamont S, Brunero S: Personality disorder prevalence

and treatment outcomes: a literature review. Issues Ment Health Nurs 2009;30:631-367.

Langbehn DR, Pfohl BM, Reynolds S, et al.: The Iowa

Personality Disorder Screen: development and preliminary validation of a brief screening interview. J Pers Disord 1999;13:75-89.

Loranger AW: International Personality Disorders Examination Manual. Odessa, FL, Psychological Assessment Ressources, 1999.

Mann AH, Raven P, Pilgrim J, et al.: An assessment of the Standardized Assessment of Personality as a screening instrument for the International Personality Disorder Examination: a comparison of informant and patient assessment for personality disorder. Psychol Med 1999;29:985-989.

Millon T, Davis R, Millon C: Manual for the MCMIIII. Minneapolis, National Computer Systems, 1997.

Moosbrugger H, Kelava A: Testtheorie und Fragebogenkonstruktion. Heidelberg, Springer, 2007.

Moran P, Leese M, Lee T, et al.: Standardised Assess-

ment of Personality - Abbreviated Scale (SAPAS): preliminary validation of a brief screen for personality disorder. Br J Psychiatry 2003;183:228-232.

Morse JQ, Pilkonis PA: Screening for personality dis-

orders. J Pers Disord 2007;21:179-198.

Murphy JM, Berwick DM, Weinstein MC, et al.: Per-

formance of screening and diagnostic tests. Arch Gen Psychiatry 1987:44:550-555.

Newton-Howes G, Tyrer P, Johnson T: Personality

disorder and the outcome of depression: meta-analysis of published studies. Br J Psychiatry 2006;188: 13-20.

Nurnberg HG, Martin GA, Somoza E, et al.: Identify-

ing personality disorders: towards the development of a clinical screening instrument. Compr Psychiatry 2000;41:137-146.
Oldham JM, Morris LB: The personality self-portrait: Why you think, work, love, and act the way you do. New York, Bantam Books, 1990.

Oldham JM, Morris LB: Wie gut kennen Sie sich selbst? Psychologie Heute 1992;19:20-29, 52-59.

Oldham JM, Morris LB: Ihr Persönlichkeitsselbstportrait. Warum Sie genauso denken, lieben und sich verhalten, wie sie es tun. Franfurt am Main, Verlag Dietmar Klotz, 2008.

Pfohl B, Blum N, Zimmermann M: The Structured Interview for DSM-IV Personality Disorders (SIDP-IV). Iowa-City, University of Iowa, Dep. of Psychiatry, 1995

Pilkonis PA, Kim Y, Proietti JM, et al.: Scales for personality disorders developed from the Inventory of Interpersonal Problems. J Pers Disord 1996;10: 355-369.

Rustenbach SJ: Metaanalyse: Eine anwendungsorientierte Einführung. Bern, Huber, 2003.

Samuels J, Eaton WW, Bienvenu III J, et al.: Preva-

lence and correlates of personality disorders in a community sample. Br J Psychiatry 2002;180:536542.

Schöttke H: Das Persönlichkeitsselbstportrait nach Oldham - Das Instrument und erste Ergebnisse. Vortrag präsentiert beim 1. Treffen der Poliklinischen Ambulanzen Universität Osnabrück und TU Braunschweig, Osnabrück, 2006.

Smith TL, Klein MH, Benjamin LS: Validation of the

Wisconsin Personality Disorders Inventory with the SCID-II. J Pers Disord 2003;17:173-187.

Thuo J, Ndetei DM, Maru H, et al.: The prevalence of

personality disorders in a Kenyan inpatient sample. J Pers Disord 2008;22:217-220.

Van Horn E, Manley C, Leddy D, et al.: Problems in

developing an instrument for the rapid assessment of personality status. Eur Psychiatry 2000;15:29-33. 